\title{
HISTORY EFFECT IN FATIGUE CRACK GROWTH UNDER MIXED MODE LOADING CONDITIONS
}

\author{
P.Y. Decreuse, S. Pommier, L. Gentot, S. Pattofatto \\ LMT-Cachan (ENS Cachan/CNRS/UPMC/UniverSud Paris), 61, avenue du Président Wilson, \\ 94235 Cachan, France
}

\begin{abstract}
Plastic deformation within the crack tip region introduces internal stresses that modify subsequent behaviour of the crack and are at the origin of history effects in fatigue crack growth. Consequently, fatigue crack growth models should include plasticity induced history effects. A model was developed and validated for mode I fatigue crack growth under variable amplitude loading conditions. The purpose of this study was to extend this model to mixed mode loading conditions. Finite element analyses are commonly employed to model crack tip plasticity and were shown to give very satisfactory results. However, if millions of cycles need to be modelled to predict the fatigue behaviour of an industrial component, the finite element method becomes computationally too expensive. By employing a multiscale approach, the local results of FE computations can be brought to the global scale. This approach consists of partitioning the velocity field at the crack tip into plastic and elastic parts. Each part is partitioned into mode I and mode II components, and finally each component is the product of a reference spatial field and an intensity factor. The intensity factor of the mode I and mode II plastic parts of the velocity fields, denoted by $d \rho_{I} / d t$ and $d \rho_{I I} / d t$, allow measuring mixed-mode plasticity in the crack tip region at the global scale. Evolutions of $d \rho_{\mathrm{I}} / \mathrm{dt}$ and $\mathrm{d} \rho_{\mathrm{II}} / \mathrm{dt}$, generated using the FE method for various loading histories, enable the identification of an empirical cyclic elastic-plastic constitutive model for the crack tip region at the global scale. Once identified, this empirical model can be employed, with no need of additional FE computations, resulting in faster computations. With the additional hypothesis that the fatigue crack growth rate and direction can be determined from mixed mode crack tip plasticity $\left(d \rho_{\mathrm{I}} / \mathrm{dt}\right.$ and $\mathrm{d} \rho_{\mathrm{II}} / \mathrm{dt}$ ), it becomes possible to predict fatigue crack growth under I/II mixed mode and variable amplitude loading conditions. To compare the predictions of this model with experiments, an asymmetric four point bend test system was set-up. It allows applying any mixed mode loading case from a pure mode I condition to a pure mode II. Initial experimental results showed an increase of the mode I fatigue crack growth rate after the application of a set of mode II overload cycles.
\end{abstract}


Keywords: fatigue crack growth, mixed mode, plasticity, model, finite element analyses

\section{Nomenclature}

FE

Finite element method

LEFM Linear Elastic Fracture Mechanics

$K_{I}^{\infty}, K_{I I}^{\infty} \quad$ Mode I and mode II nominal stress intensity factors

$\underline{u}_{I}^{e}, \underline{u}_{I I}^{e} \quad$ Mode I and mode II elastic reference displacement fields

$\underline{u}_{I}^{c}, \underline{u}_{I I}^{c} \quad$ Mode I and mode II complementary reference displacement fields

$\widetilde{K}_{I}, \widetilde{K}_{I I} \quad$ Mode I and mode II pseudo-elastic intensity factors

$\rho_{I}, \rho_{I I} \quad$ Mode I and mode II plastic intensity factors

$K_{I}^{X}, K_{I I}^{X} \quad$ Centre of the elastic domain, global measure of internal stresses at crack tip.

\section{Introduction}

Crack tip plasticity is known to be at the origin of memory effects in fatigue of metallic materials [1-8], which has posed difficulties in modelling fatigue crack growth under variable amplitude loadings. Moreover, history effects are closely related to the cyclic elastic-plastic behaviour of the material [9-10], which makes the use of a universal model questionable. The application of a mode I overload delays the fatigue crack growth. The overload yields the material ahead of the crack tip creating compressive residual stresses in the overload's plastic zone. As a consequence, the efficiency of subsequent fatigue cycles is reduced and the rate of fatigue crack growth is decreased. This is commonly known as plasticity-induced crack closure [3]. These history effects are also present under variable amplitude mixed mode loading conditions [12-22].

For instance, Dahlin \& Olsson [14] performed experiments to determine the influence periodic mode II loading had on mode I cycles. They concluded that mode II cycles decrease the fatigue crack growth rate during mode I cycles. Similar observations were made by Gau \& Upul [13] for low alloyed steel. For the same type of experiments, Nayeb-Hashemi \& Taslim [18] found a transient acceleration just after the loading application.

Concerning crack growth direction, no one has yet developed a universal global criterion to 
predict the direction of the crack under mixed mode loading. There is general agreement in the literature that plasticity in the crack tip region and induced history effects modify both the growth direction and the fatigue crack growth rate. This paper aims at proposing a global model for describing the evolution of crack tip plasticity and internal stresses when non-proportional mixed mode loading conditions are encountered. In the future this model could be employed in a fatigue crack growth criterion.

Finite element methods are useful for analyzing crack tip plasticity under various loading conditions [4,9-11, 19-22]. In particular, FE analyses allow accounting for rather complex material constitutive behaviour [9,10]. However, the simulation of mode I or mixed-mode fatigue crack growth by elastic-plastic finite element computations leads to huge computation cost. In order to model service conditions in engineering applications, the computations become even more expensive, because real components often have fatigue lives of millions cycles, and cracks do not generally remain planar. The objective of the proposed methodology is to combine the precision of local finite element computations with the rapidity of a global approach [25-27].

\section{Material and experiments.}

The studied material is a S355NL steel used for marine applications. Its chemical composition is reported in Table 1 . The grains are equi-axed and their size is typically around 20 micrometers.

Table 1: Chemical composition of the S355NL steel in weight \%.

\begin{tabular}{|c|c|c|c|c|c|c|c|c|}
\hline $\mathrm{C}(\%)$ & $\mathrm{Mn}(\%)$ & $\mathrm{P}(\%)$ & $\mathrm{S}(\%)$ & $\mathrm{Si}(\%)$ & $\mathrm{Al}(\%)$ & $\mathrm{Cr}(\%)$ & $\mathrm{Cu}(\%)$ & $\mathrm{Ni}(\%)$ \\
\hline 0.12 & 1.46 & 0.016 & 0.01 & 0.44 & 0.041 & 0.02 & 0.01 & 0.01 \\
\hline
\end{tabular}

Cylindrical specimens were cut from a $16 \mathrm{~mm}$ thick metal sheet in order to characterize the elastic-plastic constitutive behaviour of the S255NL steel. Strain controlled push-pull tests were performed with increasing strain amplitude. The material displayed kinematic hardening and the isotropic hardening was negligible. The cyclic elastic-plastic behaviour of this material [29] was modelled using the Von Mises criterion and the non-linear kinematic hardening law of Armstrong and Frederick, standard in Abaqus 6.5. The material parameters used for the simulations are reported in Table 2. A reasonable agreement is found between experiments and simulations (Fig. 1), but it might be useful to improve the description of the kinematic hardening rule in the future. 
This material constitutive behaviour is used in the following for the finite element simulations of crack tip plasticity under mixed mode conditions.

Table 2: Parameters of the elastic-plastic constitutive behaviour employed for the simulations.

\begin{tabular}{|l|l|}
\hline Young's modulus (GPa) & 180000 \\
\hline Poisson's ratio & 0.3 \\
\hline Initial yield stress Ro (MPa) & 178 \\
\hline Kinematic hardening parameter C (GPa) & 100 \\
\hline Kinematic hardening rate $\gamma$ & 500 \\
\hline
\end{tabular}
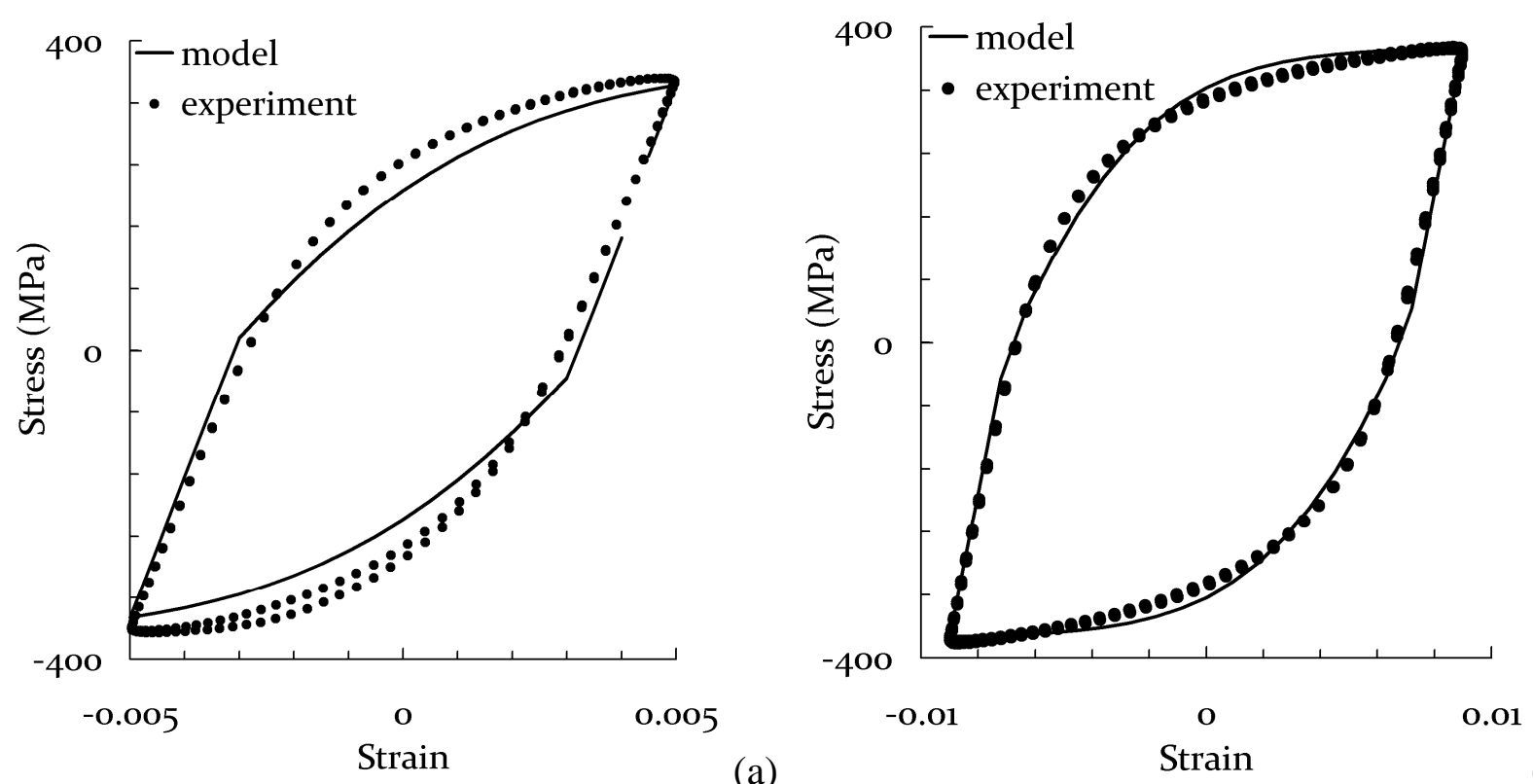

(a)

(b)

Figure 1: Strain controlled push pull test. Comparison between experimental and simulated stressstrain curves at $R_{\varepsilon}=-1$ and $\varepsilon_{\max }=0.5 \%$ (a) or $\varepsilon_{\max }=0.9 \%$ (b). Symbols: Experiments, lines: simulations.

Four point bending specimens were also cut from the same material source, with a thickness of 5 $\mathrm{mm}$, a length of $180 \mathrm{~mm}$ and a width of $30 \mathrm{~mm}$. A notch with length $8 \mathrm{~mm}$ and thickness $1.5 \mathrm{~mm}$ was created and the specimens were pre-cracked under mode I conditions with a stress ratio of 0.1 .

The specimens were mounted on a non-symmetric four point bend test system. Load was applied to the sample through roller bearings (Fig. 2). When the bearings were placed symmetrically with 
respect to the crack plane, a mode I stress intensity factor is achieved. On the contrary an antisymmetric position creates a mode II stress intensity factor. The relations between the bending moment and $K_{I}^{\infty}$, and between the shear force and $K_{I I}^{\infty}$, were determined using solutions from a fracture mechanics handbook [30]. Any intermediate position of the bearings corresponds to a given proportional mixed mode loading condition (Fig. 2). Crack extension was determined using potential drop measurements. The relation between the crack length and the potential drop was calibrated using both the initial and final crack dimensions and intermediate crack tip positions determined using digital image correlations (DIC). As a matter of fact, during the experiments images of the surface were taken periodically to determine the crack tip position and deviation of the crack growth plane.

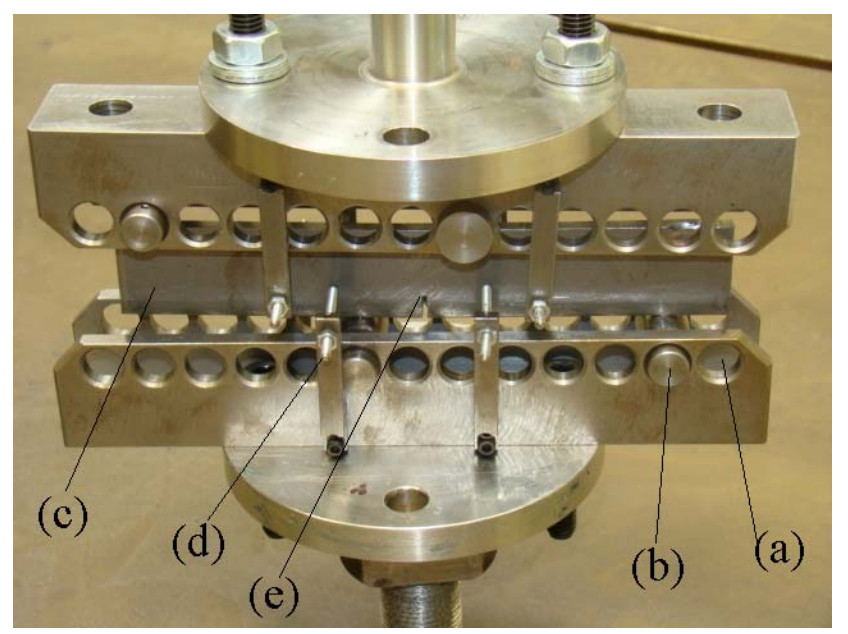

Figure 2: Non-symmetric four point bending system in an intermediate position. (a) seating for the roller bearings, (b) axles of the roller bearings adjusted to the seatings, (c) sample, (d) lateral positioning, (e) initial notch.

First, a specimen was tested to failure under pure mode I loading to generate a reference da/dN$\Delta \mathrm{K}$ curve. Second, a crack was grown under mode I conditions to a length of $12 \mathrm{~mm}$. At this point, $\Delta K_{I}^{\infty}=10 \mathrm{MPa} \sqrt{m}$. The bearings were displaced so as to apply a mode II load and the load was adjusted such that $\Delta K_{I I}^{\infty}=20 \mathrm{MPa} \sqrt{m}$ and $\mathrm{R}=0.1$. No further fatigue crack growth was observed, even after 500000 mode II cycles. The conclusion of this first experiment was that a mode II loading of $\Delta K_{I I}^{\infty}=20 \mathrm{MPa} \sqrt{m}$ was not sufficient to promote fatigue crack growth after mode I loading of $\Delta K_{I}^{\infty}=10 \mathrm{MPa} \sqrt{m}$. 
Third, a crack was grown to a length of $12 \mathrm{~mm}$ under the same mode I conditions as in the second experiment. Then the bearings were displaced to apply either one, ten or fifty mode II cycles and the load adjusted such that $\Delta K_{I I}^{\infty}=20 \mathrm{MPa} \sqrt{m}$. Then the bearings were displaced again so as to apply mode I nominal fatigue cycles, and the crack was grown again until fracture. It was observed in this case that the application of ten mode II cycles accelerated the crack (circles in Fig. 3) compared to the pure mode I fatigue crack growth experiment (thick line in Fig. 3). The increase of the fatigue crack growth rate was moderate (by about 20\%) but the recovery length was large (around $3 \mathrm{~mm}$ ). This effect is moderate but significant. The increase of the crack growth rate after 50 mode II cycles is similar to that obtained after 10 mode II cycles. That obtained after only one mode II cycle was negligible. During these experiments, a slight deviation of the crack growth plane was observed just after the application of mode II cycles. However, this deviation was neglected for the calculation of $K_{I}^{\infty}$.

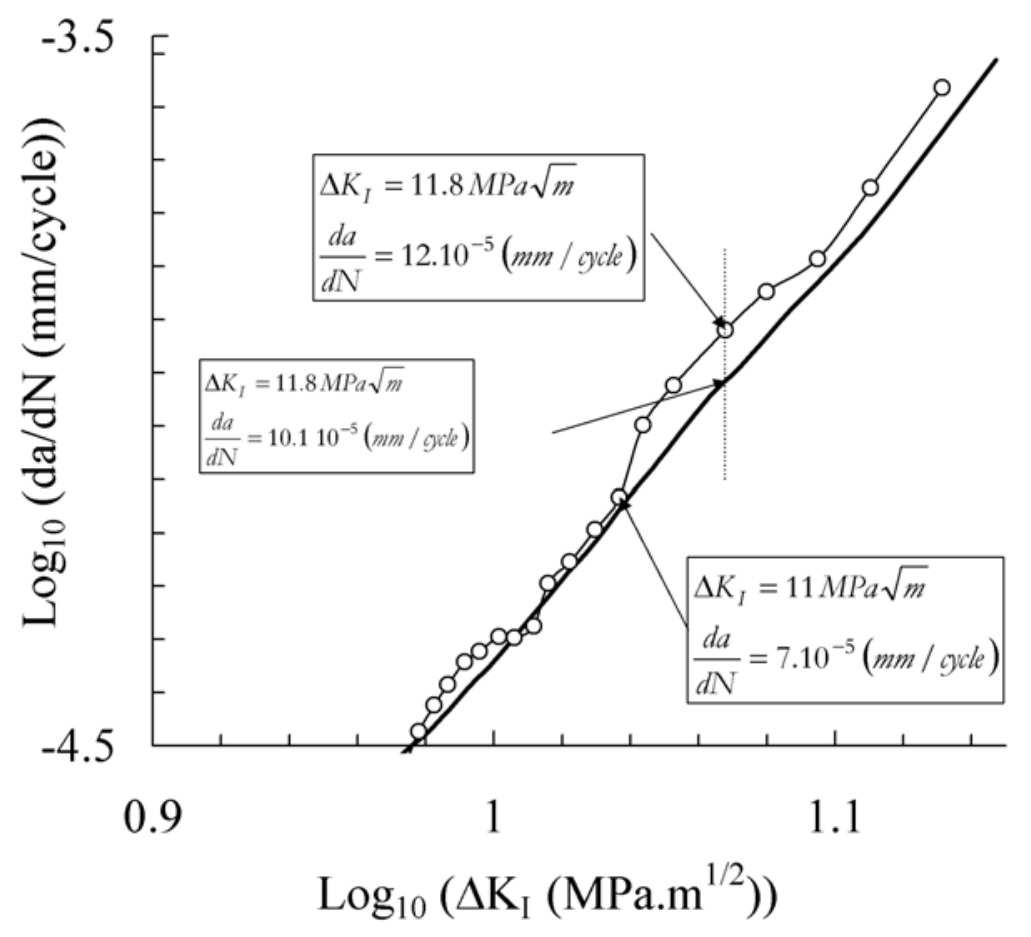

Figure 3: Crack growth rate in a Paris diagram as measured in the S355NL steel at room temperature. The thick line correspond to a reference mode I fatigue crack growth experiment with $R=0.1$. The dots corresponds to a mode I fatigue crack growth before and after the application of 10 mode II cycles with a stress ratio of 0.1 and $K_{I I}=20 \mathrm{MPa} \sqrt{m}$ 
Complementary experiments are planned in order to better characterize the mixed mode fatigue crack behaviour in the S355NL steel and related history effects. However, it can be concluded from this very first set of experiments that, for the experimental conditions tested, mode II cycles do not promote fatigue crack growth, but are at the origin of a history effect on mode I fatigue crack growth.

However, this history effect is detrimental, while various authors [14, 15, 21, 22] have found a retardation effect of a mode II cycle on mode I fatigue crack growth. In particular, Dahlin \& Olsson [14] made different experiments to study the influence of periodic mode II loading on mode I fatigue crack growth in a low alloyed steel, according to the stress ratio R of mode I cycles. First, among five different experimental conditions, only one shows an acceleration of $12 \%$ of the crack growth rate. In this case, the stress ratio and $\Delta K_{I I}$ were high $\left(R=0.58, \Delta K_{I I}=30 \mathrm{MPa} . \mathrm{m}^{1 / 2}\right)$ compared with our experimental conditions $\left(\mathrm{R}=0.1, \Delta \mathrm{K}_{\mathrm{I}}=10 \mathrm{MPa} \cdot \mathrm{m}^{1 / 2}\right)$. The other experiments were performed with a stress ratio comparable to that used in our experiments $(\mathrm{R}=0.1)$. Contrary to the experimental results in Fig.3, Dahlin \& Olsson [14] observed a decrease of the crack growth rate, regardless of the number of periodic mode II loadings.

Quite the opposite, Nayeb-Hashemi and Taslim [18] obtained only a transient acceleration of the crack after the application of a single mode II cycle, without any retardation. Their results are similar to those plotted in Fig. 3.

As a conclusion, predicting history effects in mixed mode conditions is not straightforward. History effects are suspected to be a function of the material, the stress ratio, the overload ratio, etc... Therefore, having a method to predict history effects under mixed mode conditions will be useful to design interesting loading schemes and to setup the experiment to elucidate suspected effects. In the following, such a method is developed and employed to simulate the evolution of crack tip plasticity for loading conditions equivalent to that used in the experiments (Fig. 3).

\section{Modelling}

Previously, a model was developed and validated for mode I fatigue crack growth under variable amplitude loadings conditions [25,26]. It was able to model fatigue crack growth including plasticity induced history effects. A natural evolution for this model should be to extend it to mixed mode loading conditions. Many authors [11-18] have researched this open problem, and all agree that plasticity induced history effects in mixed mode conditions modify both the growth direction 
and the growth rate of a fatigue crack.

Moreover, it was shown by Sander and Richards [21,22] and Doquet et al. [19], respectively, that suitable FE computations allow successful prediction of the crack growth rate [21,22] and direction [19] under non-proportional mixed mode conditions.

As a matter of fact, in mixed mode conditions, as in mode I conditions, cyclic plasticity within the crack tip region lies at the origin of internal stresses that superimpose on the applied stresses and modify the behaviour of the crack. The FE method allows determining local loading conditions within the crack tip region that can then be used in a local crack growth criterion. For instance, Doquet et al [19] used elastic-plastic FE computations to determine stresses and strains around the crack tip for sequential mode I / mode II cycles. The average stress and strain fields over a critical distance from the crack tip were then computed and used to determine the plane for which the fatigue damage rate per cycle is the highest. The damage criterion was, for instance, a function of the normal stress amplitude. Other criteria are also used according to the material and the damage mechanism. It allows determination of the crack propagation plane. This approach was shown to predict successfully the conditions for which the fatigue crack bifurcates for sequential mode I / mode II loading conditions.

Unfortunately, these approaches remain limited in practice because of the huge costs of the implementation of FE models an of elastic-plastic cracked body and of their analysis in fatigue. Moreover, in an industrial context, a fatigue crack growth model may have to handle millions of cycles, which imposes the use of simplified methods. Therefore, a global model, which would be easier to handle than a local one, is desired for this problem.

\subsection{The multiscale approach}

The main advantage of using the FE method is that it makes possible the use of complex material constitutive behaviours required to compute the details of the evolution of plastic deformation, residual stresses, stress range, etc... within and around the crack tip region for a stationary or moving crack tip $[9,10]$.

The local FE results are then brought to the global scale using a multiscale approach tailored for crack problems [25-27]. The global data generated by the post-treatment of FE computations are used to identify a global cyclic elastic-plastic empirical model (denoted by $f$ ) for the crack tip 
region. This model is incremental to facilitate its use in variable amplitude fatigue. For instance, in mode I, the global plasticity rate, $d \rho_{I} / d t$, within the crack tip region is a function of the nominal loading rate $d K_{I}^{\infty} / d t$ and the state of a set of internal variables [25-27]. In addition, a proportional growth law between the rate $d a / d t$ of production of cracked area per unit length of the crack front and $d \rho_{I} / d t$, allows prediction of the crack growth rate. Finally, the model is a set of ten scalar partial differential equations.

The procedure to identify the parameters in the model $f$ requires knowledge of the material cyclic elastic-plastic behaviour. It is performed using the FE method and is fully automatic. The identification of the parameter in the growth law requires the results of a constant amplitude fatigue crack growth experiment. More details about the global model development can be found in [25].

Once identified, the global model can be used to predict the fatigue crack growth rate for variable amplitude loadings schemes. It accounts for plasticity induced history effects and is cheap in computation cost. The objective is now to extend this approach for non-proportional I/II mixed mode problems.

\subsubsection{Basis}

For plane problems, the velocity field within the crack tip region in I/II mixed mode conditions is partitioned into mode I and mode II components that correspond to the symmetric and antisymmetric parts of the displacement field with respect to the crack plane, respectively. In LEFM, the velocity field is then approximated by the product of spatial reference fields $\left(\underline{u}_{I}^{e}\right.$ and $\left.\underline{u}_{I I}^{e}\right)$ and their nominal stress intensity factors rates $\left(\dot{K}_{I}^{\infty}\right.$ and $\left.\dot{K}_{I I}^{\infty}\right)$ (Eq. 1).

$$
\underline{v}(P)-\underline{v}(t i p) \approx \dot{K}_{I}^{\infty} \underline{u}_{I}^{e}(P)-\dot{K}_{I I}^{\infty} \underline{u}_{I I}^{e}(P)
$$

In order to extend this approach to an elastic-plastic behaviour, two additional spatial reference fields $\left(\underline{u}_{I}^{c}\right.$ and $\left.\underline{u}_{I I}^{c}\right)$ and their intensity factor rates $\left(\dot{\rho}_{I}\right.$ and $\left.\dot{\rho}_{I I}\right)$ are used to describe the velocity field in the crack tip region (Eq. 2).

$$
\underline{v}(P)-\underline{v}(t i p) \approx \dot{\widetilde{K}}_{I} \underline{u}_{I}^{e}(P)+\dot{\rho}_{I} \underline{u}_{I}^{c}(P)+\dot{\widetilde{K}}_{I I} \underline{u}_{I I}^{e}(P)+\dot{\rho}_{I I} \underline{u}_{I I}^{c}(P)
$$


The additional spatial reference fields $\left(\underline{u}_{I}^{c}\right.$ and $\left.\underline{u}_{I I}^{c}\right)$ are constructed to be non-dimensional such that the intensity factor rates ( $\dot{\rho}_{I}$ and $\dot{\rho}_{I I}$ ) are equivalent to the rate of the crack tip opening displacement and the crack tip sliding displacement $(d C T O D / d t, d C T S D / d t)$, respectively.

At this point, the velocity field in the crack tip region is fully defined by a set of global variables, $\underline{v}\left(t_{i p}\right)$, the crack tip velocity, and the intensity factor rates of the elastic and plastic parts of each mode $\left(\dot{\widetilde{K}}_{I}, \dot{\widetilde{K}}_{I I}, \dot{\rho}_{I}, \dot{\rho}_{I I}\right)$.

Velocity fields $\underline{v}(P)$ for stationary or moving cracks in elastic-plastic conditions are calculated using the finite element method for various load histories. A POD based routine is then used to approach $\underline{v}(P)$ either by Eq. 2 (elastic approximation) or by Eq. 3 (elastic-plastic approximation) and to determine the related errors (Eq. 3 and 4)

$$
\begin{aligned}
& C_{e}=\left(\sum_{P_{i} \in D}\left(\underline{v}\left(P_{i}\right)-\underline{v}(t i p)-\dot{K}_{I}^{\infty} \underline{u}_{I}^{e}\left(P_{i}\right)-\dot{K}_{I I}^{\infty} \underline{u}_{I I}^{e}\left(P_{i}\right)\right)^{2}\right) /\left(\sum_{P_{i} \in D}\left(\underline{v}\left(P_{i}\right)-\underline{v}(t i p)\right)^{2}\right) \\
& C_{e p}=\left(\sum_{P_{i} \in D}\left(\underline{v}\left(P_{i}\right)-\underline{v}(t i p)-\dot{\widetilde{K}}_{I} \underline{u}_{I}^{e}\left(P_{i}\right)-\dot{\rho}_{I} \underline{u}_{I}^{c}\left(P_{i}\right)-\dot{\widetilde{K}}_{I I} \underline{u}_{I I}^{e}\left(P_{i}\right)-\dot{\rho}_{I I} \underline{u}_{I I}^{c}\left(P_{i}\right)\right)^{2}\right) / \sum_{P_{i} \in D}\left(\underline{v}_{i}\left(P_{i}\right)-\underline{v}(t i p)\right)^{2}
\end{aligned}
$$

During a time increment, the crack tip region (D) is assumed to behave essentially elastically if the elastic approximation of $\underline{v}(P)$ is just as precise as the elastic-plastic one $\left(C_{e}-C_{e p} \leq C_{t h}\right)$. On the contrary, if the approximation of $\underline{v}(P)$ is improved by the use of two additional spatial reference fields $\left(\underline{u}_{I}^{c}\right.$ and $\left.\underline{u}_{I I}^{c}\right)$ and their intensity factor rates $\left(\dot{\rho}_{I}\right.$ and $\left.\dot{\rho}_{I I}\right)$, the crack tip region plastically deforms.

\subsubsection{Implementation}

2D finite element simulations were performed using Abaqus 6.5. Linear plane strain elements were used to mesh a quasi infinite sheet $(20 \mathrm{~m} \times 20 \mathrm{~m})$ containing a central crack with a length of $2 \mathrm{a}=60 \mathrm{~mm}$. The mesh was highly refined around the crack tip with a mesh size of $3 \mu \mathrm{m}$ at $10 \mu \mathrm{m}$ from the crack tip (Figure 4). The "crack tip region” was defined as the set of elements at a distance below $1 \mathrm{~mm}$ from the crack tip. 

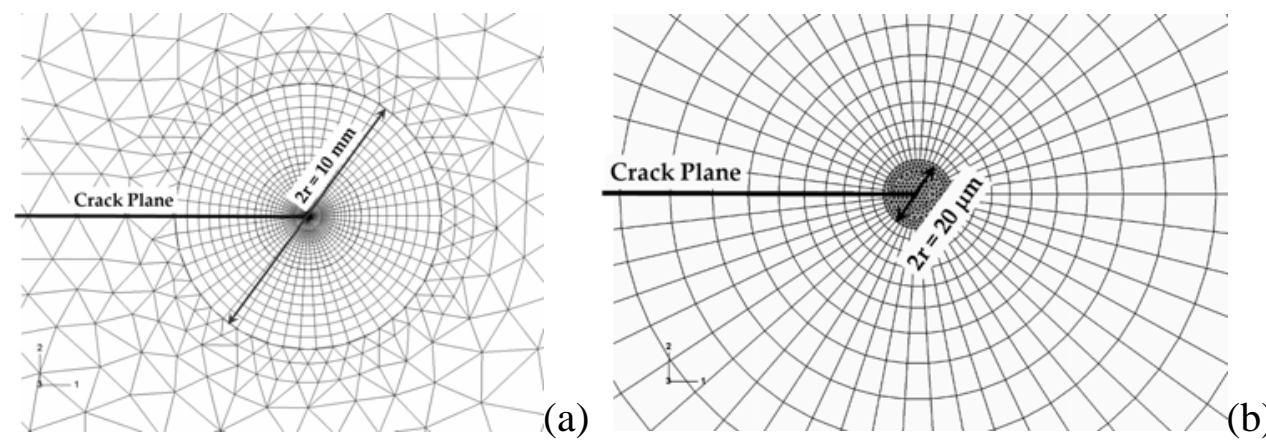

Figure 4: Finite elements mesh, (a) increasing mesh refinement around the crack tip, (b) detail of the mesh around the crack tip.

The first step of the procedure consists in determining the two reference elastic fields, $\underline{u}_{I}^{e}$ and $\underline{u}_{I I}^{e}$, from elastic finite element computations for which the nominal stress intensity factors were $K_{I}^{\infty}=1 M P a \sqrt{m}$ and $K_{I I}^{\infty}=1 M P a \sqrt{m}$, respectively.

The complementary fields, $\underline{u}_{I}^{c}$ and $\underline{u}_{I I}^{c}$, were then determined using elastic-plastic FE computations and the material constitutive law (Table 2),. To construct $\underline{u}_{I}^{c}$ for instance, the following procedure was used. A monotonic mode I tensile phase is simulated using the FE method. The displacement increment $\underline{\Delta u}(P)=\underline{u}\left(P, t_{2}\right)-\underline{u}\left(P, t_{1}\right)$ is computed at each point of the model during the last time increment of that loading phase. Then the displacement field $\underline{\Delta u}(P)$ during that increment is projected onto the mode I elastic reference field, which allows computation of the variation of its intensity factor during the increment :

$$
\widetilde{K}_{I}\left(t_{2}\right)-\widetilde{K}_{I}\left(t_{1}\right)=\frac{\int_{D} \Delta \underline{u}(P) \cdot \underline{u}_{I}^{e}(P)}{\int_{D} \underline{u}_{I}^{e}(P) \cdot \underline{u}_{I}^{e}(P)}
$$

The remainder of the displacement field (see Eq. 1) is then computed:

$$
\underline{\Delta u}^{R}(P)=\underline{\Delta u}(P)-\left(\widetilde{K}_{I}\left(t_{2}\right)-\widetilde{K}_{I}\left(t_{1}\right)\right) \underline{u}_{I}^{e}(P)
$$

And then made non-dimensional so $\dot{\rho}_{I}$ could be read as the average relative mode I displacement between the crack faces, in micrometers: 


$$
\underline{u}_{I}^{c}(P)=\underline{u}^{\mathrm{R}}(P) \cdot \frac{\int_{r=10 \mu m}^{r=1 m m} 1 \mu m}{\int_{r=10 \mu m}^{r m m}\left(u_{y}^{\mathrm{R}}(r, \theta=\pi)-u_{y}^{\mathrm{R}}(r, \theta=-\pi)\right)}
$$

The same procedure was employed in mode II. Once these preliminary computations have been performed for a given material constitutive equation, $\underline{u}_{I}^{e}, \underline{u}_{I I}^{e}, \underline{u}_{I}^{c}$ and $\underline{u}_{I I}^{c}$ are defined and can be employed for any cyclic mixed mode loading.

In the following, various mixed mode conditions are simulated using the FE method. For each time increment $\left(t_{n+1}-t_{n}\right)$, the computed displacement field during the time increment $\left[\underline{u}\left(P, t_{n+1}\right)-\underline{u}\left(P, t_{n}\right)\right]$ is stored and partitioned into mode I $\left[\underline{u}_{I}\left(P, t_{n+1}\right)-\underline{u}_{I}\left(P, t_{n}\right)\right]$ and mode II components $\left[\underline{u}_{I I}\left(P, t_{n+1}\right)-\underline{u}_{I I}\left(P, t_{n}\right)\right]$ (e.g. symmetric and anti-symmetric parts). Then the four intensity factor variations during the increment are determined, as shown in equations 8 to 11 :

$$
\begin{aligned}
& \widetilde{K}_{I}\left(t_{n+1}\right)-\widetilde{K}_{I}(t)=\frac{\int_{D}\left[\underline{u}_{I}\left(P, t_{n+1}\right)-\underline{u}_{I}\left(P, t_{n}\right)\right] \cdot \underline{u}_{I}^{e}(P)}{\int_{D} \underline{u}_{I}^{e}(P) \cdot \underline{u}_{I}^{e}(P)} \\
& \rho_{I}\left(t_{n+1}\right)-\rho_{I}(t)=\frac{\int_{D}\left[\underline{u}_{I}\left(P, t_{n+1}\right)-\underline{u}_{I}\left(P, t_{n}\right)\right] \cdot \underline{u}_{I}^{c}(P)}{\int_{D} \underline{u}_{I}^{c}(P) \cdot \underline{u}_{I}^{c}(P)} \\
& \widetilde{K}_{I I}\left(t_{n+1}\right)-\widetilde{K}_{I I}(t)=\frac{\int_{D}\left[\underline{u}_{I I}\left(P, t_{n+1}\right)-\underline{u}_{I I}\left(P, t_{n}\right)\right] \cdot \underline{u}_{I I}^{e}(P)}{\int_{D} \underline{u}_{I I}^{e}(P) \cdot \underline{u}_{I I}^{e}(P)}
\end{aligned}
$$

$$
\rho_{I I}\left(t_{n+1}\right)-\rho_{I I}(t)=\frac{\int_{D}\left[\underline{u}_{I I}\left(P, t_{n+1}\right)-\underline{u}_{I I}\left(P, t_{n}\right)\right] \cdot \underline{u}_{I I}^{c}(P)}{\int_{D} \underline{u}_{I I}^{c}(P) \cdot \underline{u}_{I I}^{c}(P)}
$$

The mean least square errors, $C_{e}$ and $C_{e p}$, between the computed displacement field and the approximation in Eq. 1 and 2 are also calculated at each increment. The error $C_{e p}$ associated with 
the elastic-plastic approximation was always below 10\% whatever the mode mixity and the history provided that the equivalent stress intensity factor used in the simulations is reasonable (e.g. below $50 \mathrm{MPa} \cdot \mathrm{m}^{1 / 2}$ for this steel). This procedure was fully automated using a python script in Abaqus and requires a few minutes to post-treat FE simulations.

\subsubsection{Results}

Various loading histories were simulated. A few examples are given in Fig. 5 to illustrate how $\rho_{I}$ and $\rho_{I I}$ (Fig. 5b), that measure crack tip plasticity at the global scale, evolve according to the mixed mode loading conditions (Fig. 5a). Three steps are applied in each case. The same loading conditions are applied during the two first steps (Fig. 5a). For the first case, though $K_{I}^{\infty}$ is kept constant during the last step, both $\rho_{I I}$ and $\rho_{I}$ increase when $K_{I I}^{\infty}$ increases. For the second case, both $\rho_{I I}$ and $\rho_{I}$ vary when $K_{I I}^{\infty}$ is kept constant and $K_{I}^{\infty}$ increases. On the contrary, in the fourth case, $\rho_{I}$ is nearly constant, while both $K_{I I}^{\infty}$ and $K_{I}^{\infty}$ vary. And finally in the third case, during the last step of the computation, $K_{I I}^{\infty}$ and $K_{I}^{\infty}$ are both decreasing but $K_{I}^{\infty}$ remains positive. In this case, though $K_{I}^{\infty}$ is decreasing, $\rho_{I}$ increases. In other words, the crack plastically blunts during the unloading phase.
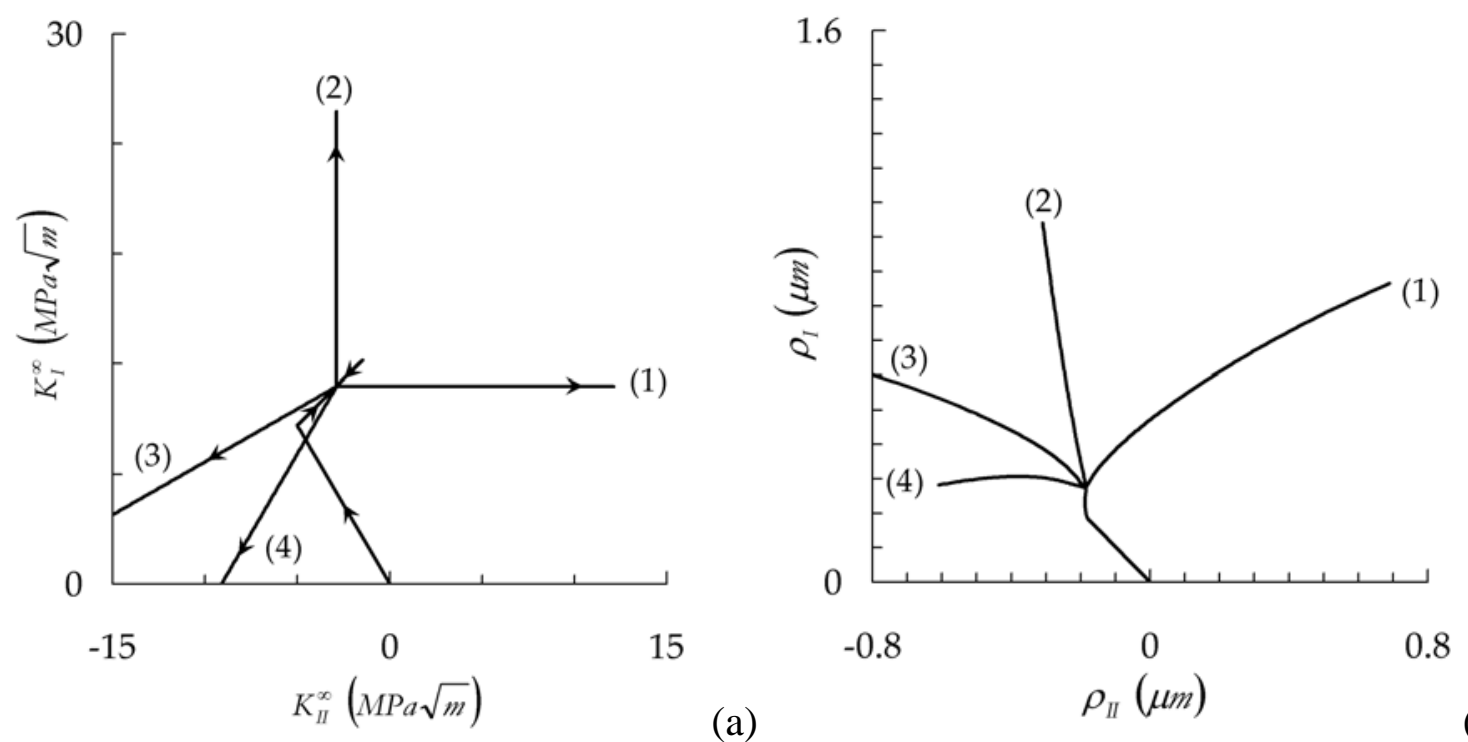

(a)

(b)

Figure 5: (a) Loading histories applied to the FE model. (b) Plastic intensity factors $\rho_{I}$ and $\rho_{I I}$. 
These results are qualitatively consistent with experimental and numerical results from Dahlin and Olsson [14] who have shown that focus should not be placed only on "ranges". The behaviour of a crack in mixed-mode conditions differs significantly whether or not a static mode I load is superimposed with a cyclic mode II load [14].

\subsection{Model}

The aim is now to find a set of simple variational evolution equations that would reproduce FE results such as those in Fig. 5, and, once identified for a given material, could then be employed to predict mixed mode plasticity at the crack tip in preference to expensive FE computations.

For this purpose a global cyclic elastic-plastic model for the crack tip region is built. It contains a yield criterion, a flow rule and a hardening rule. It is analogous to a plasticity model except that it applies to cracks. Details about this model can be found in [31].

\subsubsection{Yield criterion}

Using the multiscale approach detailed in $§ 3.1$ it is possible to apply a given load history to the crack, and then explore radiating loading directions (Fig. 6a). In each direction, the "yield" threshold is determined as the point above which the approximation of the velocity field by Eq. 2 is better than an elastic approximation (Eq. 1). When the yield point is reached, $\dot{\rho}_{I}$ and $\dot{\rho}_{I I}$ are non negligible. It is therefore possible to construct numerically the elastic domain for the crack tip region (Fig. 6b) and its evolution during loading (Fig. 6c).

Using the FE method, it is found that the size and the shape of this domain remains the same during loading (Fig. 6c). The elastic domain is roughly an ellipsis in a $K_{I I}^{\infty}$ and $K_{I}^{\infty}$ diagram. However, its center evolves during loading (Fig. 6c). The displacement of the center of the elastic domain is due to the growth of internal stresses within the crack tip region because of the development of a plastic strain gradient at crack tip. The center of this domain, denoted by $K_{I I}^{X}$ and $K_{I}^{X}$, is therefore introduced as an internal variable in the model. $K_{I I}^{X}$ and $K_{I}^{X}$ stand, at the global scale, for the internal stress field within the crack tip region. 

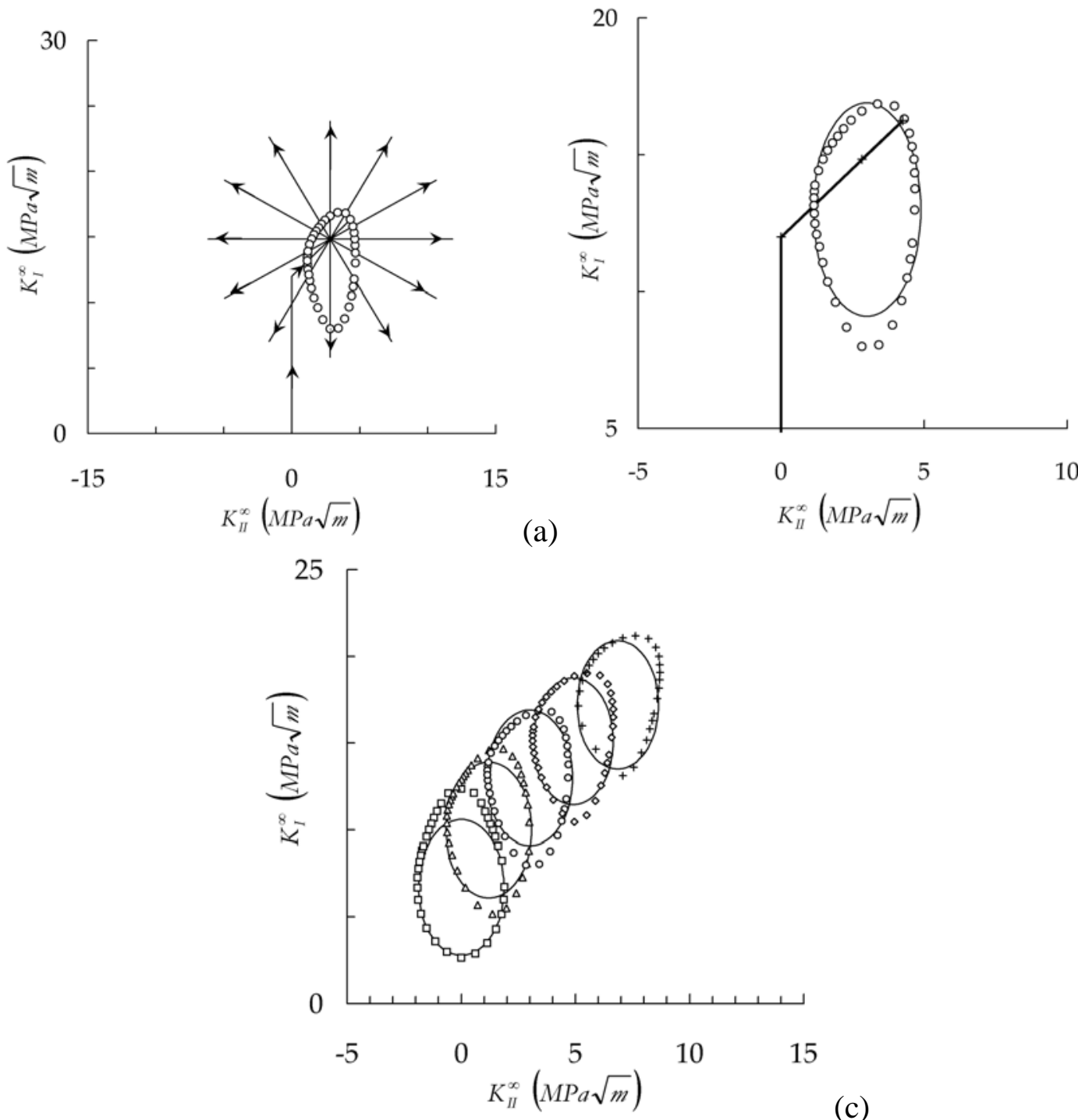

(b)

Figure 6: Construction of the global yield locus of the crack tip region. (a) typical loading histories applied during FE computations. (b) dots: yield points as determined using the FE method for each radial loading direction. Solid lines: yield locus as defined from the generalized Von Mises criterion (Eq. 12). (c) Yield locus evolution during loading (dots : from FE, solid lines : Eq. 12).

About 2 hours of FE computations are required to construct each yield surface.

Inside the elastic domain, the crack tip region behaves essentially elastically. Therefore the Westergaard equations apply (provided that $K_{I}^{\infty}-K_{I}^{X}$ and $K_{I I}^{\infty}-K_{I I}^{X}$ are used) and can be used to determine an analytical expression of the elastic shear energy inside any circular domain around the crack tip. At the local scale the material obeys the Von Mises yield criterion, which stems from a critical shear elastic energy density. Therefore, a critical shear elastic energy criterion in the crack tip region was expected to be a suitable yield criterion at the global scale. The generalized Von Mises yield criterion $f_{Y}$ obtained by this method matches almost perfectly the FE results (lines in 
Fig. 6c) and it sums up into a very simple equation (Eq. 12). The elastic domain, defined by $f_{Y}$, has a size controlled by the adjustable parameter $K_{I}^{Y}$ and a fixed aspect ratio $K_{I I}^{Y} / K_{I}^{Y}$ function of the Poisson's ratio of the material (Eq. 13).

$$
\begin{aligned}
& f_{Y}\left(K_{I I}^{\infty}, K_{I}^{\infty}, K_{I I}^{X}, K_{I}^{X}\right)=\left(K_{I}^{\infty}-K_{I}^{X}\right)^{2}+\left(\frac{K_{I}^{Y}}{K_{I I}^{Y}}\right)^{2}\left(K_{I I}^{\infty}-K_{I I}^{X}\right)^{2}-\left(K_{I}^{Y}\right)^{2} \\
& \frac{K_{I I}^{Y}}{K_{I}^{Y}}=\frac{\sqrt{7-16 v+16 v^{2}}}{\sqrt{19-16 v+16 v^{2}}}, \quad \text { if } v=0.3: K_{I I}^{Y} / K_{I}^{Y} \approx 0.48
\end{aligned}
$$

\subsubsection{Flow rule, hardening rule and equations of the model.}

A flow rule and a hardening rule were then introduced. The normality flow rule was adopted (in a $\mathrm{G}_{\mathrm{I}}, \mathrm{G}_{\mathrm{II}}$ diagram rather than in a $\mathrm{K}_{\mathrm{I}}, \mathrm{K}_{\mathrm{II}}$ ). This flow rule determines the direction of $d \rho_{I}$ and $d \rho_{I I}$ (Eq. 17) once the yield surface is reached.

Finally, we also assume as a hardening rule, that the displacement of the center of the yield surface coincides with the plastic flow direction, which yields : $d K_{I I}^{X}=p d \rho_{I I}$ and $d K_{I}^{X}=p d \rho_{I}$.

To summarize briefly, the first elements of a global variational formulation of the mixed-mode cyclic elastic-plastic behaviour of the crack tip region were gathered. First, at the global scale, plasticity in the crack tip region was represented by the rates $d \rho_{I I}$ and $d \rho_{I}$. A yield function $f_{Y}$ was introduced (Eq. 14), which is a function of the nominal applied stress intensity factors, $K_{I}^{\infty}$ and $K_{I I}^{\infty}$, and of two internal variables, $K_{I}^{X}$ and $K_{I I}^{X}$, that define the current position of the center of the yield surface and, at the global scale, describe the internal stress fields.

$$
\begin{aligned}
& f_{Y}\left(G_{I I}, G_{I}\right)=\left|G_{I}\right|+\frac{G_{I}^{Y}}{G_{I I}^{Y}}\left|G_{I I}\right|-G_{I}^{Y} \\
& \text { where } G_{I I}=\operatorname{sign}\left(K_{I I}^{\infty}-K_{I I}^{X}\right) \frac{\left(K_{I I}^{\infty}-K_{I I}^{X}\right)^{2}}{E^{*}} \text { and } G_{I}=\operatorname{sign}\left(K_{I}^{\infty}-K_{I}^{X}\right) \frac{\left(K_{I}^{\infty}-K_{I}^{X}\right)^{2}}{E^{*}}
\end{aligned}
$$

The global plastic strain rate is assumed to obey the normality flow rule, which is expressed as follows: 
If $f<0$ or if $(f=0 \& d f<0)$ then $: d \rho_{I}=d \rho_{I I}=d K_{I I}^{X}=d K_{I}^{X}=0$

Else : $d \rho_{I I}=d \lambda \frac{\partial f}{\partial G_{I I}}$ and $d \rho_{I}=d \lambda \frac{\partial f}{\partial G_{I}}$

And the evolution equations for the center of the yield surface are as follows, $\mathrm{R}$ being a “material” parameter :

$$
d K_{I}^{X}=p d \lambda \frac{\partial f}{\partial G_{I}}, d K_{I I}^{X}=p d \lambda \frac{\partial f}{\partial G_{I I}} \text { where } p=\frac{R}{2} \frac{\left(\left|\frac{\partial f}{\partial G_{I}}\right|+\frac{G_{I I}^{Y}}{G_{I}^{Y}}\left|\frac{\partial f}{\partial G_{I I}}\right|\right)}{\left(\left|\frac{\partial f}{\partial G_{I}}\right|+\frac{G_{I}^{Y}}{G_{I I}^{Y}}\left|\frac{\partial f}{\partial G_{I I}}\right|\right)}
$$

During plastic deformation, the yield criterion $f=0$ is always fulfilled, which imposes that $d f=0$. This last equation allows determining $d \lambda$, since $d G_{I}$ and $d G_{I I}$ are function of $d K_{I}^{X}$ and $d K_{I I}^{X}$, which are themselves function of $d \lambda$. In practice, $d \lambda$ is determined numerically using the radial return algorithm.

\subsubsection{Validation}

At this point, loading histories were simulated both using the finite element method and the global model (Fig. 7) to check the validity of the flow rule and the hardening rule. Since plasticity is constrained within the crack tip region, either stress controlled or displacement controlled boundary conditions can be used to impose nominal stress intensity factors for FE computations. In the present case, displacement controlled boundary conditions are used.

First, finite element computations were performed to validate the hardening rule. The evolutions of the center of the elastic domain were determined for four loading cases (Fig. 7). It is worth underlining that getting each single position of the center of the elastic domain requires 12 finite element computations (see Fig. 6a). Those positions are plotted as full symbols in Fig. 7a. It was found that the evolutions of $K_{I}^{X}$ and $K_{I I}^{X}$, calculated using the global model, matched almost perfectly that obtained using the FE method (Fig. 7 a). The model did not reproduce as well the evolutions of $\rho_{I}$ and $\rho_{I I}$ (Fig. 7 b), but the main trends are captured (directions and amplitudes). 

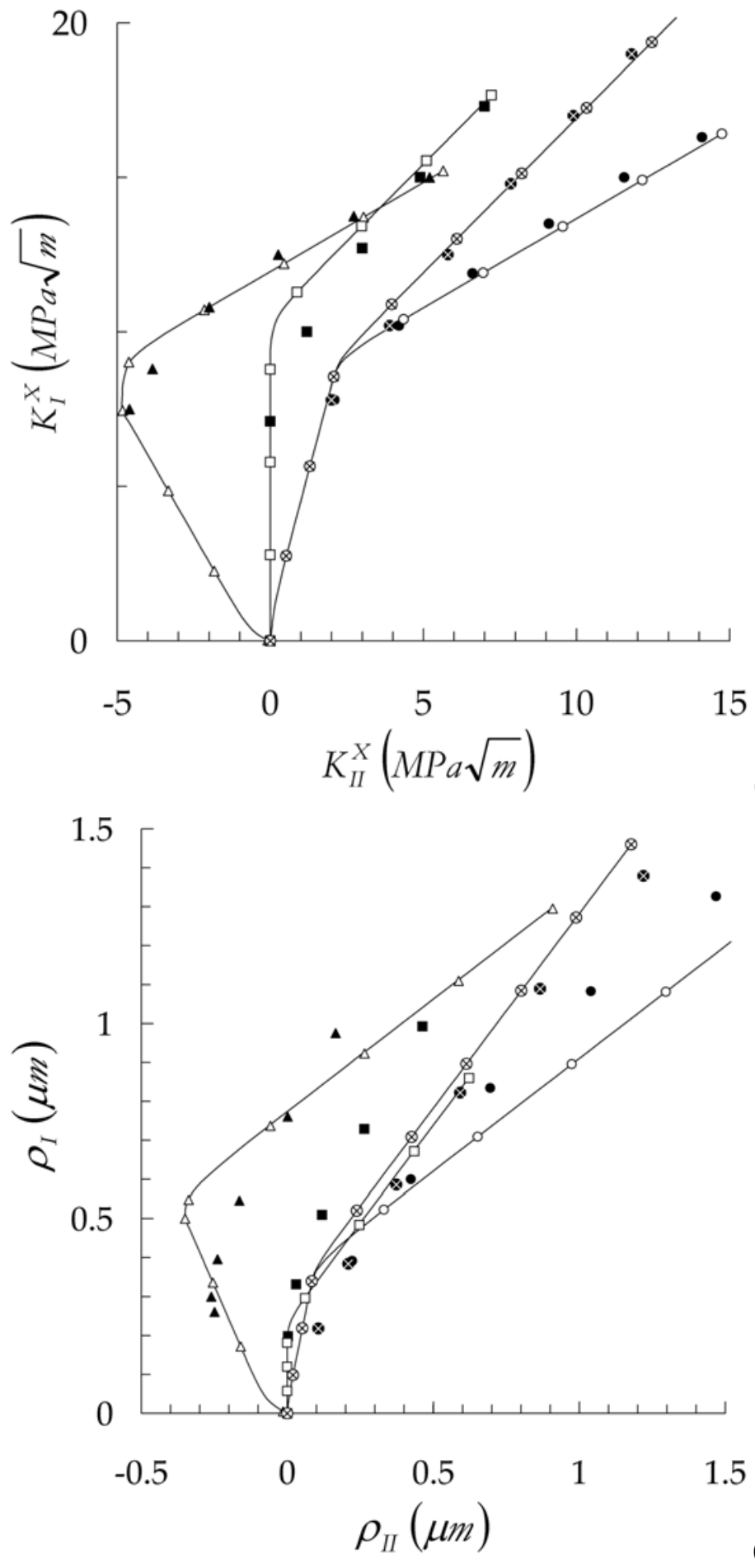

(a)

(b)

Figure 7: (a) Positions of the centres of the yield surfaces and (b) plastic intensity factors $\rho_{I}$ and $\rho_{\text {II }}$ for the same loading histories, as determined either using FE computations (full symbols) or using the global variational model (Eq. 11-25) (lines and empty symbols).

A second validation case is plotted in Fig. 8. Five out of phase mixed mode loading cycles (Eq. 19) were applied, with a stress intensity factor range of $20 \mathrm{MPa} \cdot \mathrm{m}^{1 / 2}$. 


$$
K_{I I}^{\infty}(t)=K_{o} \sin (\omega t) \quad \text { and } \quad K_{I}^{\infty}(t)=K_{o}(1+\cos (\omega t)) \quad \text { with } \quad K_{o}=10 M P a \sqrt{m}
$$

The evolutions of $\rho_{I I}$ and $\rho_{I}$ were calculated either using the FE method or using the global model. The hysteresis loop stabilizes after the first cycle. In Fig. 8, the fifth cycle only is reported. The nominal applied stress intensity factor is plotted in Fig 8(a). The evolution of $\rho_{I I}$ and $\rho_{I}$ calculated using the finite element method and the global model are plotted in Fig. 8 (b) as full symbol and lines, respectively. The global plastic intensity factor ranges $\Delta \rho_{I I}$ and $\Delta \rho_{I}$ are reasonably predicted by the model. It is worth mentioning that though the nominal stress intensity factor loading path is circular, the plastic intensity factor path is not circular. The shape of the cycle is successfully predicted, including the inclination of the ellipsis. However, as in Fig. 7 (b), the global model does not coincide precisely with the results of FE computations.
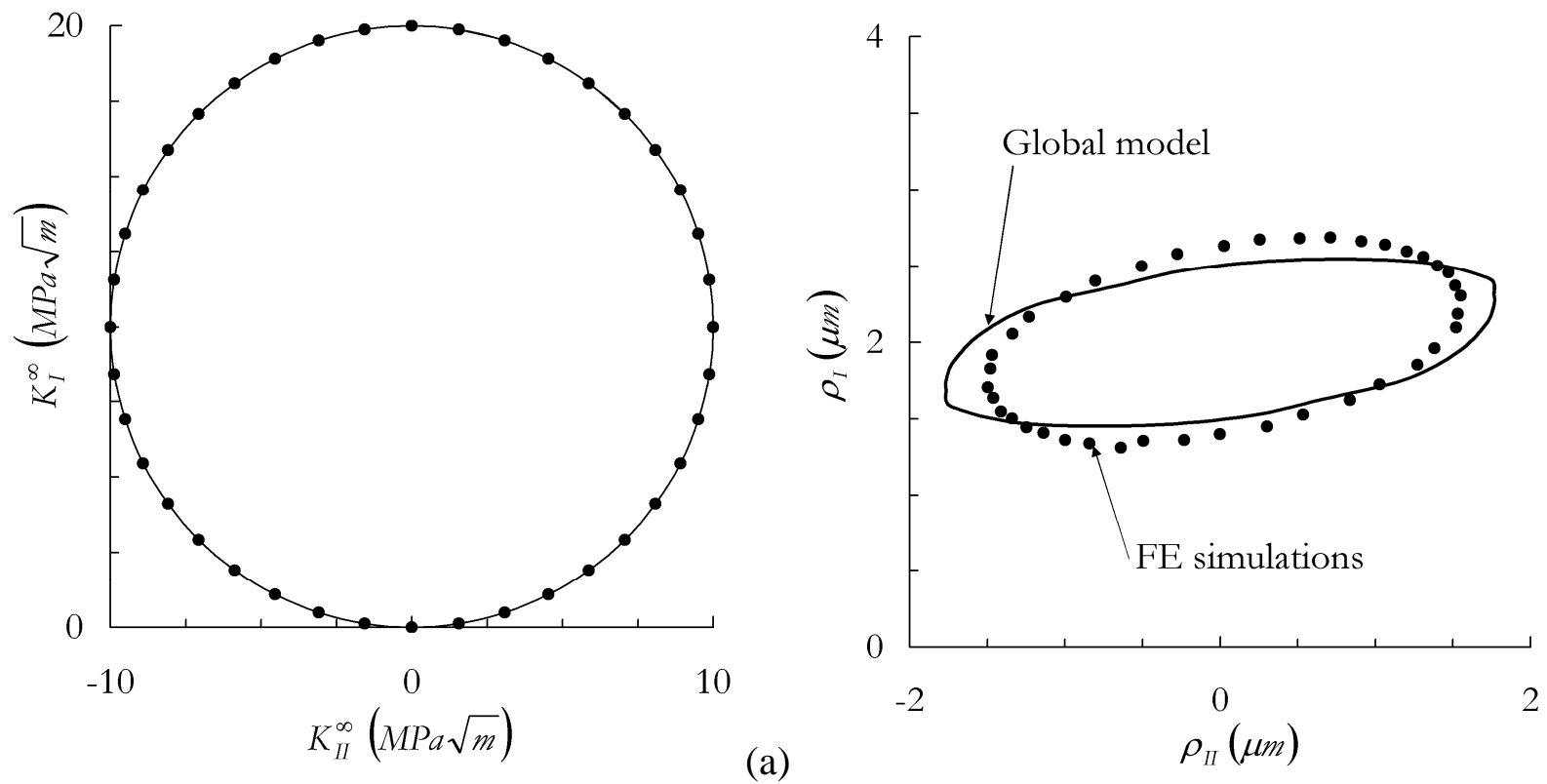

Figure 8: (a) Applied nominal stress intensity factors (b) plastic intensity factors $\rho_{I I}=C s t+\int d \rho_{I I}$ and $\rho_{I}=C s t+\int d \rho_{I}$ from FE computations (black circles) or from the global model (line).

\subsection{Application and discussion}

The global model was then used to simulate the loading histories employed in the experiments. For mode I cycles $\Delta K_{I}=10 M P a \sqrt{m}$ and $\mathrm{R}=0$, and for mode II cycles, $\Delta K_{I I}=20 M P a \sqrt{m}$ and $\mathrm{R}=0$. First, 170 mode I cycles were simulated using the global model. Second, 150 mode I cycles were 
simulated, followed by 10 mode II cycles and 10 mode I cycles. The evolutions of $\rho_{I I}$ and $\rho_{I}$ during the last 10 mode I cycles are plotted in Fig. 9 for the two cases.
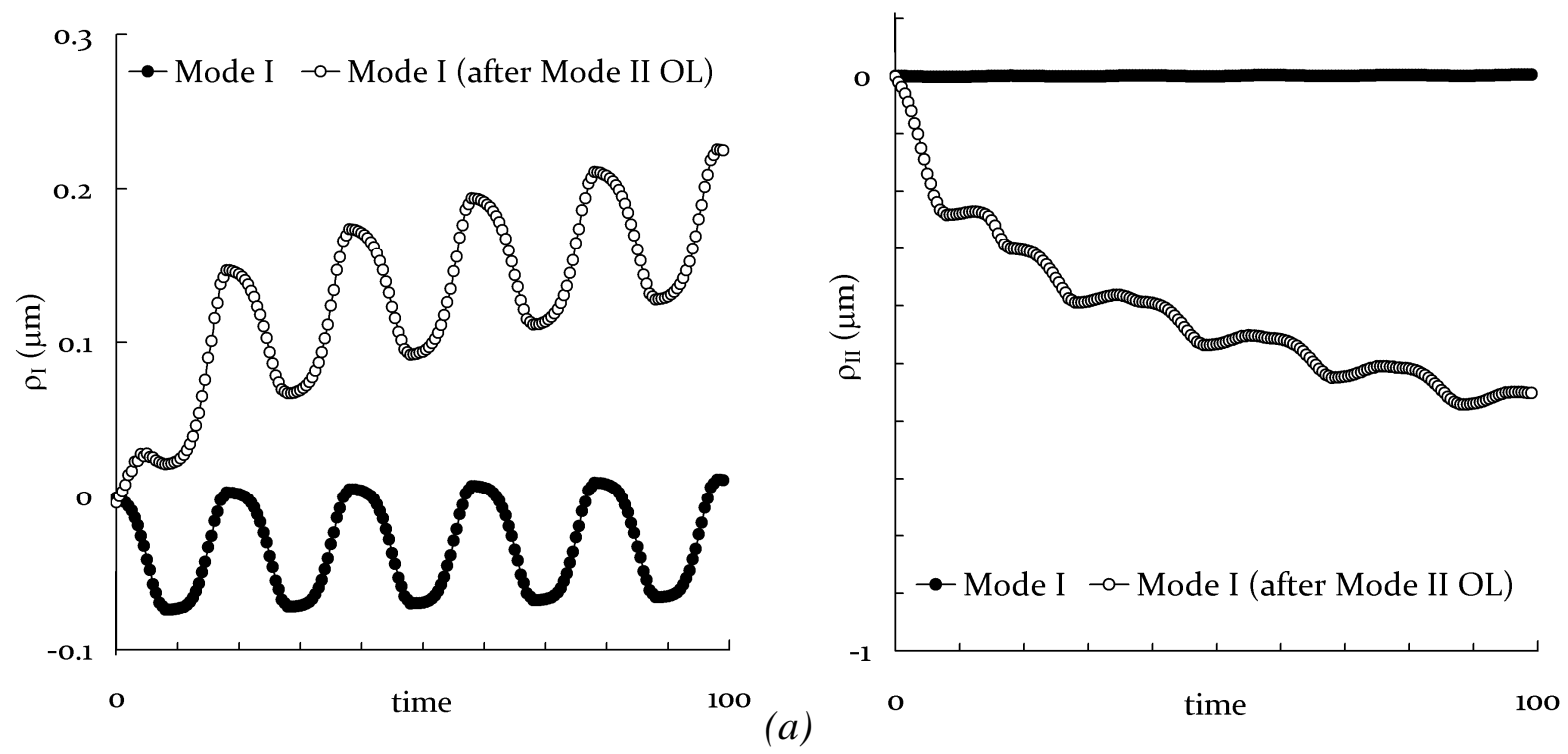

(a)

Figure 9: Simulations of crack tip plastic deformation for the loading cases used in the experiments

(Fig. 3). The black circles correspond to 10 mode I cycles after 160 mode I cycles. The empty circles correspond to 10 mode I cycles after 10 mode II overloads and 150 mode I cycles.

Calculated evolutions using the global mode (a) $\rho_{I}$, (b) $\rho_{I I}$.

It is observed that, after the application of mode II overloads, the mode I plastic intensity factor range $\left(\Delta \rho_{I}\right)$ is increased by about a factor 2 . If the crack growth rate is proportional to the plastic intensity factor range, an increase of the fatigue crack growth rate after the application of the mode II overloads should be predicted using this model. This result is consistent with experimental observations. Furthermore, the mode I plastic intensity factor $\rho_{I}$ increases progressively. Since this model is incremental ( $\rho_{I}=C s t+\int d \rho_{I}$ ), the value of $\rho_{I}$ alone is not significant. However, the progressive increase of $\rho_{I}$ should tend to blunt the crack and remove any crack closure effect.

\section{Conclusions and prospects}

The aim of this study was to establish a mixed-mode crack propagation model including history effects and to validate it using suitably chosen experiments. For this purpose, mixed mode fatigue 
crack growth experiments were setup and showed that the studied material (a S355 NL steel used in marine applications) shows history effects in mixed mode fatigue crack growth. In the future it is planned to study in a systematic manner the fatigue crack growth resistance in this material under complex mixed-mode conditions.

Also, the first elements of a global mixed-mode plasticity model for the crack tip region were gathered. Since it is recognized that plasticity is at the origin of large history effects, a global cyclic elastic-plastic model for the crack tip region is useful for predicting mixed mode fatigue crack growth when variable amplitude conditions are encountered. This model is dedicated to be used in elastic analyses of cracked structures and should account for memory effects inherited from the non-linear behaviour of the material within the crack tip region. It can be used for instance in crack tip XFEM elements or in cohesive zones.

First, a multiscale approach was used to bring the results of detailed elastic plastic FE analyses from the local scale to the global scale. For this purpose, the velocity field in the crack tip region was approximated by the product of spatial reference fields, known a priori, and of their intensity factors. This approach is classical in LEFM. It was merely extended to the case of elastic-plastic material behaviours by introducing two additional spatial reference fields ( $\underline{u}_{I}^{c}$ and $\left.\underline{u}_{I I}^{c}\right)$ and their intensity factors ( $\rho_{I}$ and $\rho_{I I}$ ) to account for plastic deformation within the crack tip region. Such an approximation was shown to be reasonably precise (less than $10 \%$ error), whatever the modemixity and the load history. Then during any loading history, the four intensity factor rates ( $\dot{\widetilde{K}}_{I I}, \dot{\widetilde{K}}_{I}, \dot{\rho}_{I I}, \dot{\rho}_{I}$ ) are determined by projecting the computed velocity field onto the basis of reference spatial fields that were constructed for all $\left(\underline{u}_{I}^{e}, \underline{u}_{I I}^{e}, \underline{u}_{I}^{c}\right.$ and $\left.\underline{u}_{I I}^{c}\right)$.

In certain cases, approximating the velocity field by using only the "elastic” reference spatial fields $\left(\underline{u}_{I I}^{e}, \underline{u}_{I}^{e}\right)$ is just as precise as using the "enriched" approximation. In such cases, the crack tip region is considered as behaving elastically.

These FE results were used to define a global elastic plastic constitutive behaviour for the crack tip region. It includes: a yield criterion, a flow rule and a kinematic hardening evolution equation and sums up into a set of five scalar differential equations. 
From the results of FE computations it was shown that an elastic domain can be defined for the crack tip region, which evolves during loading. This domain can be modelled by a yield criterion (generalization of the Von Mises criterion). During loading, the center of the yield locus moves because of the growth of internal stresses. Therefore, two internal variables $\left(K_{I I}^{X}, K_{I}^{X}\right)$ were introduced to define the current position of this yield locus, and describe at the global scale these internal stresses. The normality flow rule was adopted for this problem and allows predicting $\dot{\rho}_{I I}$ and $\dot{\rho}_{I}$ from the current state of the variables of the model and from the loading rate. And finally, the center of the yield locus moves along the plastic flow direction.

This model computes the evolutions of the current origin of the elastic domain for the crack tip region with good precision, compared with the FE method. The advantage is that the computations using this model, unlike FE computations, are very fast and efficient. The prediction of $\dot{\rho}_{I I}$ and $\dot{\rho}_{I}$ remains to be improved, but the flow directions and the order of magnitude of the flow rate are correctly reproduced.

In the future, this model should be enriched. First, the evolution law of the crack closure as a function of $\dot{\rho}_{I I}$ and $\dot{\rho}_{I}$ needs to be defined. Second, how the internal variables in this model evolve when the crack grows or deviates needs to be determined. This part can be done using the same approach, based on FE computations. Finally, a crack growth criterion, to predict the fatigue crack growth rate under general mixed mode non-proportional and variable amplitude loading conditions from the knowledge of $\dot{\rho}_{I I}$ and $\dot{\rho}_{I}$, needs to be developed. For this purpose fatigue crack growth experiments should be performed under complex loading conditions.

\section{Acknowledgements}

This work is supported by the French National Research Agency (ANR) through the project MIXMODFATFIS (No. BLAN06-1_134492) and by the Research Agency of the French Ministry of Defense (DGA).

\section{References}

1. Suresh, S., (1991), Fatigue of Materials, Cambridge University Press, Cambridge.

2. Mc Clung., R.C., (1996), proc. Fatigue 96, 6th International Fatigue Congress, Berlin FRG, 6-10 May 1996.

3. $\quad$ Elber, W., (1971), ASTM STP 486, pp. 230-242. 
4. $\quad$ Fleck, N.A., Newman, J.C., (1988) ASTM STP 982, pp. 319-341

5. $\quad$ Newman, J.C., (1981), ASTM STP 748, 1981, pp. 53-84

6. $\quad$ Schijve, J., (1973), Engineering Fracture Mechanics, (5) pp. 269-280.

7. $\quad$ McMillan, J.C., Pelloux, R.M.N., (1967), ASTM STP 415, pp. 505-532

8. $\quad$ Forman, R.G., Kearney, V.E. , Engle, R.M. (1967), Journal of basic Engineering, Vol 89, pp. 459-64.

9. $\quad$ Pommier, S., (2002), Plane strain crack closure and cyclic hardening, Engineering Fracture Mechanics, vol. 69, pp. 25-44.

10. Pommier, S., (2003), Cyclic plasticity and variable amplitude fatigue, International Journal of Fatigue, vol. 25 pp. 983-997.

11. Pommier, S., (2006), proc. Plasticity'06 - 12th International Symposium on Plasticity and its Current Applications. Halifax (Canada). 17-22 July 2006

12. Srinivas, V., Vasudevan, P., (1993), Study of the influence of mixed mode overload on mode I fatigue crack propagation, Int J Vessels Piping, Vol. 56, pp. 409-417.

13. Gao, H., Upul, S.F. (1996), Effect of non-proportional overloading on fatigue life. Fatigue Fract Eng Mater Struct, Vol. 19, pp. 1197-1206.

14. Dahlin, P., Olsson, M. (2008), Fatigue crack growth - Mode I cycles with periodic Mode II loading, Int. J., Fatigue, Vol. 30, pp. 931-941.

15. Dahlin, P., Olsson, M. (2002), The effect of plasticity on incipient mixed mode fatigue crack growth, Fat. Fract. Engng. Mat. Struct., Vol. 26, pp. 577-588.

16. Bold, P.E., Brown, M.W., Allen, R.J., (1992), A review of fatigue crack growth in steels under mixed mode I and II loading. Fatigue Fract Eng Mater Struct, Vol. 15, pp. 965-977.

17. Wong, S.L., Bold, P.E., Brown M.W., Allen, R.J., (2000) Fatigue crack growth rates under sequential mixed-mode I and II loading cycles. Fatigue Fract Eng Mater Struct, Vol. 23, pp. 667-674.

18. Nayeb-Hashemi, H., Taslim, M.E., (1987), Effects of the transient Mode II on the steady state crack growth in Mode I, Eng Fract Mech, Vol. 26, pp. 789-807.

19. Doquet V., Bertolino G., (2008), Local approach to fatigue cracks bifurcation, Int. J. Fat., Vol. 30 (5), pp. 942-950.

20. Doquet, V., Pommier, S., (2004), Fatigue crack growth under non-proportional mixed mode loading in ferritic-pearlitic steel, Fatigue Fract Eng Mater Struct, Vol. 27, pp. 1051-1060.

21. Sander, M., Richard, H.A., (2006), Experimental and numerical investigations on the influence of the loading direction on the fatigue crack growth, Int J. Fatigue, Vol. 28, pp. 583-591.

22. Sander, M., Richard, H.A., (2005), Finite element analysis of fatigue crack growth with interspersed mode I and mixed mode overloads., Int J Fatigue, Vol. 27, pp. 905-913.

23. Erdogan, F., Sih, G.C., (1963), On the crack extension in plates under plane loading and transverse shear, J Basic Eng, Vol. 85, pp. 519-525.

24. Qian, J., Fatemi, A., (1996) Mixed mode fatigue crack growth: A literature survey, Engineering Fracture Mechanics, Vol. 55 (6), pp. 969-990. 
25. Pommier, S., Hamam R., (2007), Incremental model for fatigue crack growth based on a displacement partitioning hypothesis of mode I elastic-plastic displacement fields, F Fatigue Fract Eng Mater Struct, Vol. 30, pp. 582-598.

26. Hamam R., Pommier, S., Bumbieler, Fat., (2007), Variable amplitude fatigue crack growth, experimental results and modelling, Vol. 29, Issue: 9-11, pp. 1634-1646

27. Pommier, S., Risbet M, (2005), Time-derivative equations for fatigue crack growth in metals, Int. J. Fract., Vol. 131, pp. 79-106.

28. Li, C., (1989) Vector CTD criterion applied to mixed mode fatigue crack growth. Fatigue Fracture Engng Mater. Structures, Vol. 12, pp. 59-65.

29. Lemaître, J., Chaboche, J.L., (2004), Mécanique des Matériaux Solides, Sciences Sup, Dunod

30. Tada, H., Paris, P., (1973), The stress analysis of cracks handbook, Del research corporation, Hellertown, Pennsylvania, USA

31. Pommier, S., Lopez-Crespo, P., Decreuse, P.Y., (2008) A multiscale approach to build a global variational formulation of the mixed-mode cyclic elastic-plastic behaviour of the crack tip region, submitted to Fatigue Fract Eng Mater Struct 